\title{
Tecnura
}

\section{Estudio exploratorio del tratamiento de agua de lavado de tintas por método de electrocoagulación/electroflotación}

\section{Exploratory Study of Dye Wastewater Treatment through the Electrocoagulation/ Electroflotation Method}

\author{
María Camila García Vacaํ, César Augusto García Ubaque², \\ Juan Sebastián de Plaza Solórzano 3
}

Fecha de recepción: 29 de octubre de 2014

Fecha de aceptación: 18 de septiembre de 2015

Cómo citar: García Vaca, M. C., García Ubaque, C. A., \& de Plaza Solórzano, J. S. (2016). Estudio exploratorio del tratamiento de agua de lavado de tintas por método de electrocoagulación/electroflotación. Revista Tecnura, 20(47), 107-117. http://doi.org/10.14483/udistrital.jour.tecnura.2016.1.a09

\section{Resumen}

Las aguas de lavado de tinta se catalogan como contaminantes debido a que son sustancias en su mayoría poliaromáticas, carcinogénicas, mutagénicas y teratogénicas para gran variedad de seres vivos. Dentro de los métodos de descontaminación, la electrocoagulación/electroflotación es reconocido como un tratamiento altamente eficiente para la remoción de contaminantes disueltos en agua, y su efecto sobre colorantes ha sido estudiado y descrito. No tiene, sin embargo, aplicación industrial en Colombia en la actualidad y su lugar en los objetivos de investigación académica en el país es aún muy marginal. Por esa razón, este trabajo es un estudio exploratorio del tratamiento de un residuo industrial de tinta de imprenta por medio del método de electrocoagulación/electroflotación. Se aplicó un diseño experimental $2^{2}$ con un punto central. Se obtuvo un rango de porcentajes de remoción de DQO entre $10,43 \%$ hasta $50,93 \%$. No obstante, los resultados evidencian la posibilidad de que la descontaminación por electrocoagulación puede ser reversible, y no se observa la incidencia esperada de los factores escogidos.

Palabras clave: Electrocoagulación, electroflotación, aguas de lavado de tintas de impresión.

\begin{abstract}
Dye wastewater is considered pollutant because it may contain polyaromatic substances known as carcinogenic, mutagenic and teratogenic to a variety of living species. Within the many treatment methods, the electrocoagulation/electroflotation method has been widely studied and it is known to be very effective. However, there is currently no industrial use of it in Colombia, and it is not a priority in academic investigation either. Because of this, this work is an exploratory study of the treatment of printing dye wastewater through the electrocoagulation/electroflotation method. A $2^{2}$ with a central point experimental
\end{abstract}

Ingeniera Química, candidata a magister en Ingeniería Química Universidad de los Andes. Bogotá, Colombia. Contacto: mc.garcia149@ uniandes.edu.co

Ingeniero Civil, magister en Ingeniería Ambiental, doctor en Ingeniería. Profesor asociado Universidad Distrital Francisco José de Caldas. Bogotá, Colombia. Contacto: cagarciau@udistrital.edu.co

Ingeniero Civil, estudiante de maestría en Ingeniería Civil. Docente de la Universidad Piloto de Colombia. Bogotá, Colombia. Contacto: juan-deplaza@upc.edu.co 
design was applied. Removal percentage of OCD (Oxygen Chemical Demand) of the wastewater was obtain between $10,43 \%$ and $50,93 \%$. Nevertheless, results show that decontamination through this treatment method may be reversible, and the expected incidence of factors is not observed.

Keywords: dye wastewater, electrocoagulation, electroflotation.

\section{INTRODUCCIÓN}

Se sabe que la mayoría de los colorantes y pigmentos presentes en las tintas artificiales son de naturaleza poliaromática, por sus propiedades de absorción de luz, claves para funcionar como pigmento (Peters, 1995). No obstante, esta característica también convierte a las tintas y a los residuos acuosos de su utilización en factores altamente contaminantes para el medio ambiente, pues tienen conocido efecto carcinogénico, mutagénico y teratogénico para los seres vivos (Daneshvar, Ashassi-Sorkhabi y Tizpar, 2003).

Por esta razón, se han desarrollado diferentes métodos de descontaminación de estos compuestos que pueden clasificarse dentro de varios grupos, por ejemplo: 1) los métodos de oxidación avanzada, entre estos también aquellos asistidos por fotocatálisis (Rojas y Giraldo, 2009a, 2009b; Rojas et al., 2008), que tienen como objetivo la descontaminación directa de los compuestos en solución; 2) métodos de separación de los colorantes contaminantes del agua como: ultra y nanofiltración (Petrinić, Andersen, Šostar-Turk y Marechal, 2007) y métodos de absorción; 3) métodos de coagulación, tanto coagulación química como electrocoagulación; y también 4) métodos de electroflotación y flotación por aire disuelto (DAF).

El principio de la electrocoagulación es que los iones metálicos (tradicionalmente hierro o aluminio), al disolverse del ánodo de sacrificio, inmediatamente forman hidróxidos poliméricos; estos son los agentes coagulantes. Así, el mecanismo de desestabilización de contaminantes, suspensión de partículas y rotura de emulsiones en el proceso de electrocoagulación, se pueden resumir como sigue (Comninellis y Chen, 2010):
1. Compresión de la doble capa difusa alrededor de las especies cargadas por las interacciones de los iones generados por la oxidación del ánodo de sacrificio.

2. Neutralización de la carga de especies iónicas presentes en el agua residual por iones contrarios producidos por la oxidación del ánodo de sacrificio. Esta neutralización favorece la coagulación debido a que disminuye la repulsión electrostática de las partículas.

3. Formación de flóculos, esto es que los flóculos originados por coagulación generan una capa de sedimento fangoso que atrapa o liga partículas coloidales aún disueltas en el medio acuoso.

Adicionalmente, al hidrolizarse el agua, hay generación de burbujas de $\mathrm{O}_{2}$ y $\mathrm{H}_{2}$, que atraen los flóculos y los hacen flotar a la superficie; ese es el principio de la electroflotación. Comparada con técnicas como la flotación por aire disuelto (DAF), que trabaja mediante el mismo principio de llevar flóculos a la superficie con burbujas de gas, se cree que la electroflotación es más eficiente (Szpyrkowicz et al., 2000), por cuanto pueden producirse burbujas de menor tamaño en esta que en aquella, con una mayor área superficial por unidad de volumen, que pueden interactuar con mayor cantidad de flóculos.

Así mismo, se ha señalado la presencia conjunta de las siguientes reacciones en una celda electroquímica (Mohllah et al., 2004):

a. Reducción catódica de impurezas en el agua.

b. Descarga y coagulación de partículas coloidales.

c. Migración electroforética de los iones.

d. Electroflotación de las partículas coloidales por las burbujas de $\mathrm{O}_{2}$ y $\mathrm{H}_{2}$ producidas en los electrodos. 
e. Reducción de iones metálicos en el cátodo.

f. Otros procesos químicos y electroquímicos.

Por otro lado, se ha propuesto además que la acción descontaminadora de la electrocoagulación, va más allá de una sola separación. En vez de eso, se ha propuesto que el mecanismo primario de descomposición de los tintes es una oxidación homogénea con el "cloruro activo" generado por la electrólisis, en lugar de una descarga de contaminantes en el ánodo (Szpyrkowicz et al., 2000)

Algunas de las ventajas de la electrocoagulación con respecto a la coagulación química son (Comninellis y Chen, 2010):

1. En la coagulación química, la hidrólisis de las sales metálicas usadas como coagulantes lleva a un descenso importante del $\mathrm{pH}$, así que es necesario regular el $\mathrm{pH}$ del efluente del proceso, ya que los flóculos son altamente sensibles a las condiciones de medio ácidas; sin embargo, gracias a la hidrólisis en el proceso de electrocoagulación, se lleva a cabo una neutralización casi simultánea del pH de la solución reactante. Esto la hace efectiva en un rango mucho mayor de $\mathrm{pH}$ que la coagulación química.

2. Los flóculos generados por electrocoagulación tienden a ser más grandes, con menor contenido de agua y más resistentes a ácidos que aquellos generados por coagulación química, y su remoción se ve facilitada por el proceso de electroflotación.

3. El lodo (sedimento) formado en electrocoagulación es fácilmente descartable, pues está compuesto mayormente de óxidos e hidróxidos metálicos. Sobre todo, la electrocoagulación es una técnica poco productora de lodo.

4. No se requieren condiciones altas de temperatura o turbidez para que el método sea efectivo.

Por supuesto, la electrocoagulación tiene también desventajas que dificultan su aplicación industrial en ciertas partes del mundo, por ejemplo (Comninellis y Chen, 2010): a) los ánodos de sacrificio que se disuelven en el agua de tratamiento como resultado de la oxidación deben ser reemplazados periódicamente; b) la pasivación de los electrodos con el tiempo; c) se requiere una alta conductividad en el agua residual para el tratamiento con electrocoagulación; d) el costo de la energía eléctrica. Sin embargo, no hay duda de que este fenómeno conjunto de electrocoagulación/electroflotación es un método de descontaminación altamente efectivo para diversos tipos de contaminantes disueltos, no solo colorantes, sino una amplia gama de compuestos orgánicos. Estructuras muy variadas (Pepió y Gutiérrez-Bouzán, 2011) como tartrazina (Modirshahla, Behnajady y Kooshaiian, 2007), cristal-violeta (Bayram y Ayranci, 2010) y benzoquinona1 (Rojas et al., 2008), que forman parte de colorantes, hasta vinazas pueden ser tratadas mediante electrocoagulación/electroflotación para lograr disminución de color (Dávila, Machuca \& Marrianga, 2008, 2010). Se ha demostrado, además, que la eficiencia del método no depende de la molécula tratada como de la concentración inicial de esta (Cañizares et al., 2006).

A su vez, se han hecho numerosos estudios del método para establecer las variables que rigen el tratamiento de colorantes específicos. Estas variables son (Daneshvar, Ashassi-Sorkhabi y Tizpar, 2003; Cañizares et al., 2006; Comninellis y Chen, 2010; Can y Bayramoglu, 2010; Pepió y Gutiérrez-Bouzán, 2011):

- Concentración inicial de colorante.

- Conductividad del medio.

- Densidad de corriente.

- Tiempo de tratamiento.

- Agitación.

- Material de los electrodos.

- $\mathrm{pH}$ inicial.

- Temperatura inicial.

- Distancia entre los electrodos.

- Arreglo de múltiples electrodos.

- Periodo de inversión de la corriente de la celda electroquímica. 
Dependiendo del compuesto específico que se trate, la eficiencia del tratamiento depende en mayor o menor grado del $\mathrm{pH}$ o la temperatura. En cuanto al material de los electrodos, existen reportes de un desempeño sobresaliente de la aleación Ti/Pt-Ir (Szpyrkowicz et al., 2000), esto porque los materiales tradicionales, en especial el aluminio, alcanzan estado de pasivación, y otros materiales, como el grafito, no tienen una acción coagulante tan fuerte (Comninellis y Chen, 2010). También, se ha encontrado que el proceso es más eficiente a concentraciones iniciales bajas de colorante (Comninellis y Chen, 2010).

Por otra parte, las diferentes mediciones de descontaminación con las que se evalúa la eficiencia del método proporcionan información acerca de distintos factores de contaminación de los colorantes y las tintas. Por ejemplo, el color del agua contamina por cuanto interfiere en los procesos fotosintéticos de los organismos autótrofos en ecosistemas acuáticos, mientras que el contenido orgánico compite por el oxígeno disuelto, ya que es susceptible de oxidarse, por esto es causante de condiciones sépticas (anaerobias) en fuentes de agua. Así, en literatura se reportan tres clases de mediciones: a) porcentaje de decoloración (o disminución de color), usando espectroscopía en UV visible generalmente; b) demanda biológica y demanda química de oxígeno (DBO y DQO) y contenido orgánico total (COT), y (3) cromatografía líquida de alta eficacia (HPLC, en inglés). Las medidas de demanda de oxígeno fueron las de verdadero interés en este proyecto; pues son una medida del potencial contaminante de estos compuestos para los ecosistemas acuáticos. Pero ya que no era fácil determinar la biodegradabilidad del residuo de agua, se descartó la medida de DBO. Por eso, se realizaron medidas de DQO para los puntos del diseño experimental $2^{2}$ que se planteó con las variables que resultaron más representativas según la literatura y las pruebas preliminares. Estas variables son: la cantidad de electrolito en la solución (medido en g de $\mathrm{NaCl}$ ), que es una condición que influye directamente en la conductividad del medio, y el tiempo de tratamiento (medido en minutos).

\section{EXPERIMENTACIÓN}

\section{Materiales y métodos}

El agua de residuo de tintas fue proporcionada por la empresa El Periódico, la cual es el residuo producto de la limpieza de las máquinas de impresión: una mezcla de concentración desconocida de diversas tintas de impresión también desconocidas. El volumen de muestra fue $100 \mathrm{ml}$ por cada ensayo.

Como reactor, se utilizaron beakers de $200 \mathrm{ml}$. Debido al volumen disponible dentro del reactor, no se recurrió a dispositivos de agitación que sí están presentes en escalas mayores de un proceso de electrocoagulación/electroflotación.

El electrolito seleccionado fue $\mathrm{NaCl}$, en la forma de sal de mesa comercial de marca Refisal. La cantidad de sal ( $\mathrm{g}$ ) fue medida en una balanza modelo AJ-2200E de Shinko Denshi CO. Ltda., de precisión 0,01 g, y esta variaba según los niveles del diseño experimental $(2,50 \mathrm{~g}$ y $5,00 \mathrm{~g})$ y el puno central (3,75 g).

Se utilizaron electrodos de aluminio, láminas rectangulares de $16 \mathrm{~cm}$ de alto, $4 \mathrm{~cm}$ de ancho y 2 $\mathrm{mm}$ de grosor, con un área sumergida total de 32 $\mathrm{cm}^{2}$ tanto para el cátodo como para el ánodo. Se usó un solo par de electrodos para cada corrida, conservando una distancia de 0,7 cm entre ellos. Debido a que el paso de corriente era bajo, la

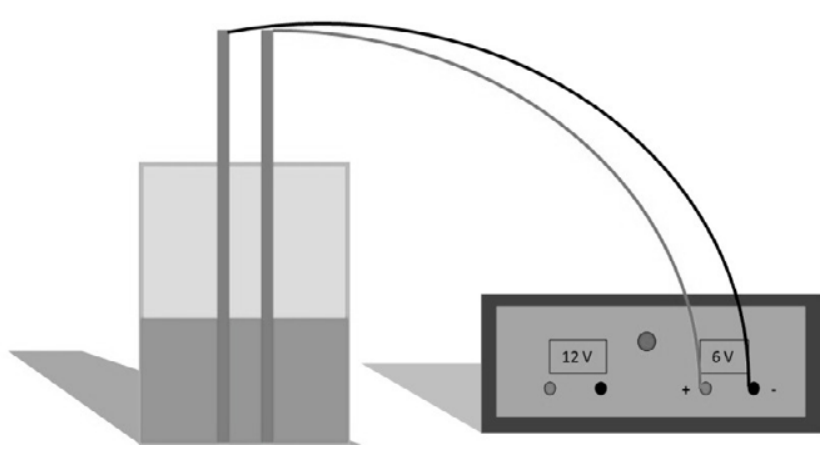

Figura 1. Esquema del montaje experimental

Fuente: elaboración propia 
pasivación de los electrodos (Comninellis y Chen, 2010) no era una limitación durante el tiempo de cada ensayo.

Antes de cada corrida, las láminas de aluminio eran pesadas y, a continuación, se sometían a un secado, con el fin de registrar su cambio de masa como efecto de la reacción electrolítica. Este secado se llevó a cabo en horno, a $50{ }^{\circ} \mathrm{C}$ y durante 15 minutos.

Luego, los electrodos se conectaban a una fuente de voltaje Kepco MPS-420-M, proporcionada por el Departamento de Ingeniería Eléctrica y Electrónica, de la Universidad de los Andes; el voltaje del circuito se fijó en $+5 \mathrm{~V}$ con una corriente de $5 \mathrm{~A}$.

El tiempo durante el cual el paso de corriente estaría activo variaba según los niveles escogidos para el diseño experimental (30 min y $15 \mathrm{~min}$ ) y el punto central (22,5 min). Este tiempo era medido con un cronómetro, y las acciones sobre el montaje (encendido y apagado de la fuente) eran todas manuales.

Una vez el tiempo de tratamiento se cumpliera para cada corrida, la fuente se apagaba y los electrodos se retiraban, se limpiaban y eran pesados una vez más. También se registró la temperatura inicial de la mezcla reactante y la final con una termocupla.

La mezcla tratada se decantaba y se almacenaba refrigerada a $4{ }^{\circ} \mathrm{C}$ por al menos $48 \mathrm{~h}$. Luego, era filtrada por gravedad con papel filtro Wahtman Cat N. ${ }^{\circ} 1001125$.

El filtrado era almacenado hasta que todas las muestras estuvieran listas para el análisis de demanda química de oxígeno.

Estos análisis se hicieron en las instalaciones del Laboratorio Ambiental del Departamento de Ingeniería Civil y Ambiental, de la Universidad de los Andes, para los cuales se tuvo en cuenta el método SM 5220 D acreditado.

El análisis estadístico del diseño experimental se desarrolló por medio del software Minitab 16@.

El diseño experimental planteado fue un $2^{2}$ con 1 punto central (tabla 1) con dos réplicas por punto:
Tabla 1. Combinación de variables y niveles para el diseño factorial $2^{2}$ con punto central

\begin{tabular}{cccc}
\hline $\begin{array}{c}\text { Punto } \\
\text { experimental }\end{array}$ & $\begin{array}{c}\text { Cantidad de } \\
\mathbf{N a C l}(\mathbf{g})\end{array}$ & $\begin{array}{c}\text { Tiempo de } \\
\text { tratamiento } \\
\text { (min) }\end{array}$ & $\begin{array}{c}\text { Notación de } \\
\text { Yates [26] }\end{array}$ \\
\hline 1 & 5,00 & 30,0 & $\mathrm{ab}$ \\
\hline 2 & 5,00 & 15,0 & $\mathrm{a}$ \\
\hline 3 & 2,50 & 30,0 & $\mathrm{~b}$ \\
\hline 4 & 2,50 & 15,0 & $(1)$ \\
\hline 5 & 3,75 & 22,5 & - \\
\hline
\end{tabular}

Fuente: elaboración propia

\section{RESULTADOS}

Tabla 2. Resultados de DQO para cada una de las muestras analizadas

\begin{tabular}{ccccc}
\hline & \multicolumn{2}{c}{ DQO $(\mathbf{m g} / \mathrm{L})$} & \multicolumn{2}{c}{$\begin{array}{c}\text { Disminución } \\
\text { de DQO }\end{array}$} \\
\hline $\begin{array}{c}\text { Punto } \\
\text { experimental }\end{array}$ & $\begin{array}{c}\text { Réplica } \\
\mathbf{1}\end{array}$ & $\begin{array}{c}\text { Réplica } \\
\mathbf{2}\end{array}$ & $\begin{array}{c}\text { Réplica } \\
\mathbf{1}\end{array}$ & $\begin{array}{c}\text { Réplica } \\
\mathbf{2}\end{array}$ \\
\hline 1 & 3632 & 2148 & 15,53 & 50,05 \\
\hline 2 & 3537 & 2110 & 17,74 & 50,93 \\
\hline 3 & 3860 & 2262 & 10,23 & 47,39 \\
\hline 4 & 2795 & 2110 & 35,00 & 50,93 \\
\hline 5 & 2205 & 2776 & 48,72 & 35,44 \\
\hline
\end{tabular}

Fuente: elaboración propia

Los resultados de DQO para las muestras tratadas se observan en la tabla 2. Las primeras réplicas de los puntos experimentales (columna "Réplica 1" para DQO $(\mathrm{mg} / \mathrm{L}))$ tienen lecturas de DQO mayores a las segundas réplicas (columna "Réplica 2"). Estadísticamente, este fenómeno introduce una variabilidad tan grande que los efectos de los factores (cantidad de $\mathrm{NaCl}$ en solución y tiempo de tratamiento) son hallados insignificantes según el análisis de Anova (tabla 3 y figura 2). Todos los $p$-values, sin excepción, son mayores que el alfa de 0,05. Además, en la figura 3, en las gráficas de los residuos, se observa un comportamiento claramente alejado de la normalidad. Esto invalida el análisis de Anova en cuanto a constructor de un modelo predictivo de la variable respuesta (Gutiérrez y De la Vara, 2004). 
Tabla 3. Anova para resultados de DQO según diseño factorial $2^{2}$ con punto central

\begin{tabular}{|c|c|c|c|c|c|}
\hline \multicolumn{6}{|c|}{ Efectos y coeficientes estimados para DQO (unidades codificadas) } \\
\hline Término & Efecto & Coef & SE Coef & $\mathbf{T}$ & $\mathbf{P}$ \\
\hline Constante & 2806,8 & 308,0 & 9,11 & 0,000 & \\
\hline Tiempo de tratamiento & 337,5 & 168,7 & 308,0 & 0,55 & 0,607 \\
\hline Cantidad de $\mathrm{NaCl}$ en solución & 100,0 & 50,0 & 308,0 & 0,16 & 0,877 \\
\hline Tiempo de tratamiento* & $-271,0$ & $-135,5$ & 308,0 & $-0,44$ & 0,678 \\
\hline \multicolumn{6}{|l|}{ Cantidad de $\mathrm{NaCl}$ en solución } \\
\hline Pt Ctral & $-316,2$ & 688,6 & $-0,46$ & 0,665 & \\
\hline$S=871,060$ & PRESS $=1$ & 74910 & & & \\
\hline R-cuad. $=12,76 \%$ R-cuad.(pre & $=0,00 \%$ & cuad.(aju & $=0,00 \%$ & & \\
\hline
\end{tabular}

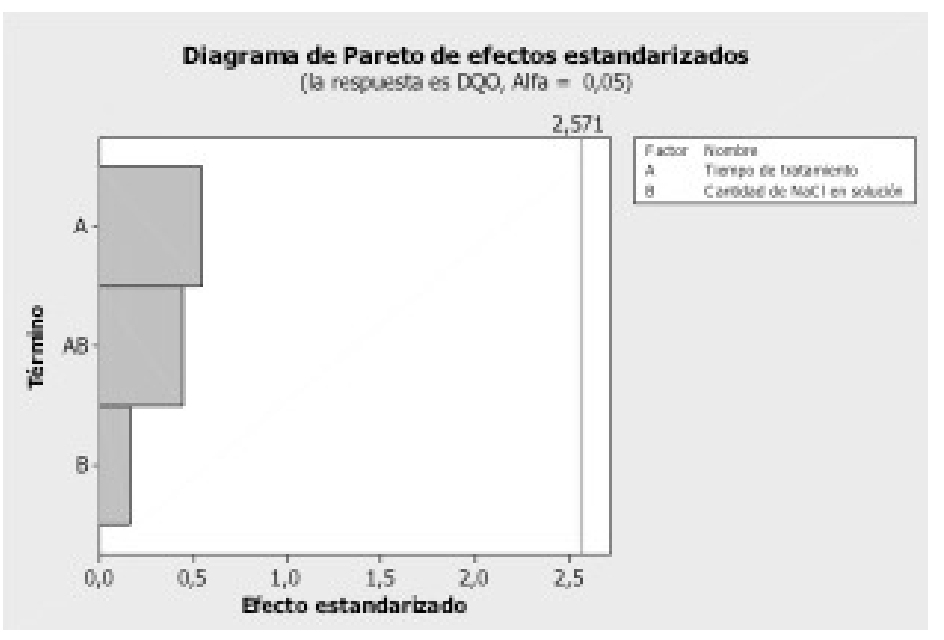

Figura 2. Pareto de efectos para DQO

Fuente: elaboración propia.

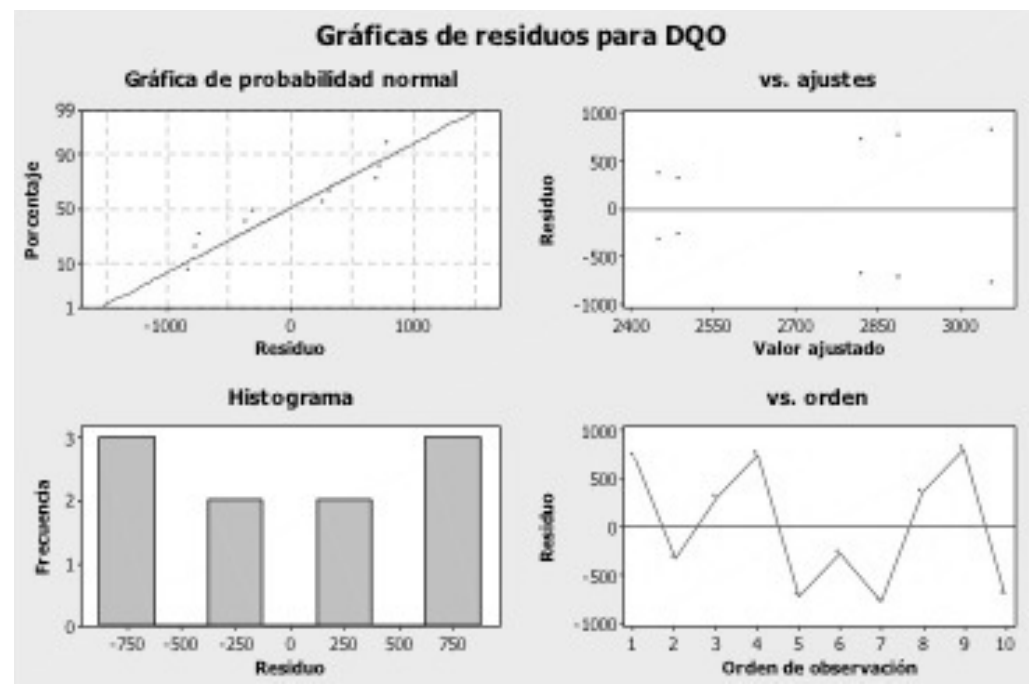

Figura 3. Gráficas de residuos para DQO

Fuente: elaboración propia. 
La explicación de este fenómeno puede estar en el funcionamiento del método mismo de DQO. La demanda química de oxígeno no es una medida directa del contenido de materia orgánica de una muestra. Es una medida del oxígeno necesario para oxidar ese contenido orgánico. Se sabe que hay materia orgánica que resiste ese tipo de oxidación y es posible que iones metálicos oxidables como el aluminio, proveniente de los ánodos de sacrificio, pudieran afectarla de estar presentes.

Por otro lado, existe un requerimiento de preservación de una muestra para DQO que se obvió debido a que se descartó su necesidad. Una muestra que vaya a analizarse con DQO debe ser lo más fresca posible y debe estar refrigerada a $4{ }^{\circ} \mathrm{C}$ y preservada con ácido sulfúrico para evitar cualquier alteración la muestra, esto porque el DQO es un análisis practicado sobre todo a aguas residuales susceptibles a actividad microbiana. Al no tener en cuenta un análisis de DBO, considerado en un momento debido a su menor costo, ya que la muestra debía ser biodegradable y era una condición que se desconocía, se descartó también cualquier posibilidad de actividad microbiana en las muestras tratadas, y por ende la necesidad de una preservación con ácido sulfúrico. $Y$ ya que las muestras no fueron tratadas simultáneamente, y se debía esperar a que todas estuvieran dispuestas para el análisis de DQO, las muestras que completaron el proceso primero (ser sometidas a electrocoagulación, ser decantadas y ser filtradas), las primeras réplicas, permanecieron más tiempo refrigeradas sin preservación que las últimas, las segundas réplicas.

La manera exacta en la que esto afectó las lecturas de DQO, o cómo preservar con ácido sulfúrico hubiera prevenido esto, no es conocida, sin embargo los resultados parecen apoyar esa tendencia no aleatoria.

Ninguna literatura disponible reporta algo así; por el contrario, se reportan modelos lineales de la descontaminación por electrocoagulación basados en la conductividad del medio y el tiempo de tratamiento (Pepió y Gutiérrez-Bouzán, 2011) estadísticamente aceptables, y también descensos de hasta
$85 \%$ en DQO como efecto del tratamiento con electrocoagulación (Comninellis y Chen, 2010).

Ya que el análisis de DQO es una medida global de materia oxidable en una muestra, no es posible saber con exactitud el efecto de una contaminación como la que se produjo o la causa de unos resultados tan atípicos.

Sin embargo, el hecho de que una muestra tratada con electrocoagulación, decantada y filtrada, registre una mayor DQO que una muestra que simplemente se mantuvo refrigerada durante el mismo tiempo, es interesante. Plantea la posibilidad de que el tratamiento de electrocoagulación es de algún modo reversible, es decir, los flóculos de hidróxido polimérico de aluminio son susceptibles de desestabilizarse y aunque en un principio atrapan materia orgánica, pueden, bajo ciertas condiciones, deshacerse y liberar parte de la materia orgánica y además moléculas metálicas de aluminio. El proceso de filtrado al que se sometieron las muestras solo se hizo para separar los flóculos y los sedimentos lodosos que se producen (figura 4, izq.), no para impedir el paso de aluminio disuelto. Así que la presencia de aluminio en los filtrados no se previno.

Cabe anotar que las lecturas más altas de DQO en la tabla 2 corresponden a los puntos experimentales con los niveles más altos de las variables, puntos en los cuales se esperaba una lectura considerablemente más baja de DQO, ya que el proceso es más eficiente con alta conductividad de medio y mayor tiempo de tratamiento según la literatura. Y estas condiciones -alta conductividad y mayor tiempo de tratamiento- originan una mayor disolución del ánodo de sacrificio (tabla 4) y, por tanto, mayor cantidad de aluminio disuelto.

Por lo general, la eficiencia del método de electrocoagulación/electroflotación, aún medida en disminución de DQO, es directamente proporcional a la disolución del ánodo de sacrificio, pues esa es su fuente de agente coagulante. Por eso, al ver el resultado del análisis de Anova para la disminución de masa del ánodo (tabla 5), se observa el comportamiento que se hubiera esperado para las lecturas de DQO de las muestras. 


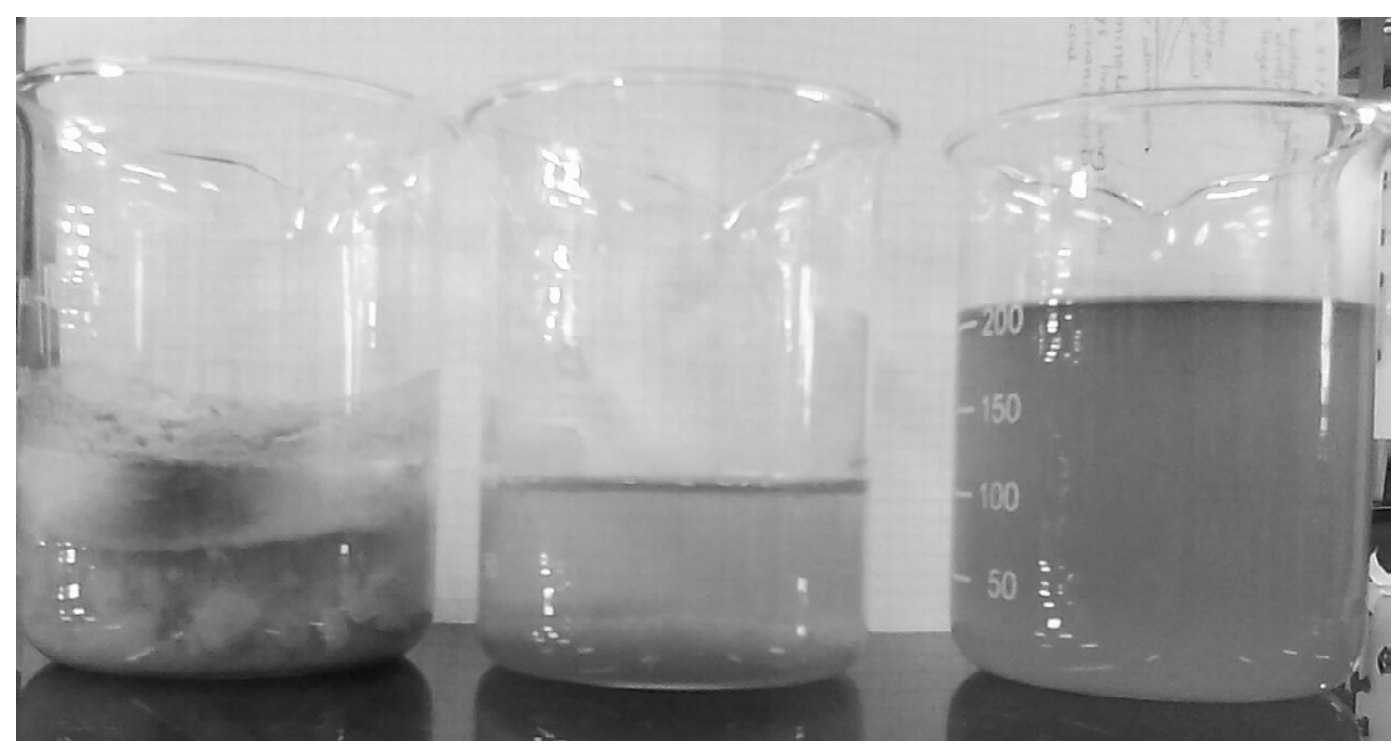

Figura 4. Estado de muestras después del tratamiento, a 25 min (izq.) y 1 min (centro), comparadas con el agua de tintas original (der.)

Fuente: elaboración propia.

Tabla 4. Disminución de la masa del ánodo de sacrificio de aluminio durante el tratamiento

\begin{tabular}{ccc}
\hline Punto experimental & Masa inicial del ánodo (g) & Disminución de masa (g) \\
\hline $1 \mathrm{a}$ & 17,11 & 0,95 \\
\hline $1 \mathrm{~b}$ & 16,78 & 1,08 \\
\hline $2 \mathrm{a}$ & 16,89 & 0,41 \\
\hline $2 \mathrm{~b}$ & 16,82 & 0,54 \\
\hline $3 \mathrm{a}$ & 16,70 & 0,71 \\
\hline $3 \mathrm{~b}$ & 16,79 & 0,73 \\
\hline $4 \mathrm{a}$ & 17,03 & 0,47 \\
\hline $4 \mathrm{~b}$ & 16,94 & 0,33 \\
\hline $5 \mathrm{a}$ & 16,57 & 0,75 \\
\hline $5 \mathrm{~b}$ & 16,85 & 0,79 \\
\hline
\end{tabular}

Fuente: elaboración propia.

Tabla 5. Anova para resultados de disminución de masa del ánodo de sacrificio según diseño factorial $2^{2}$ con punto central

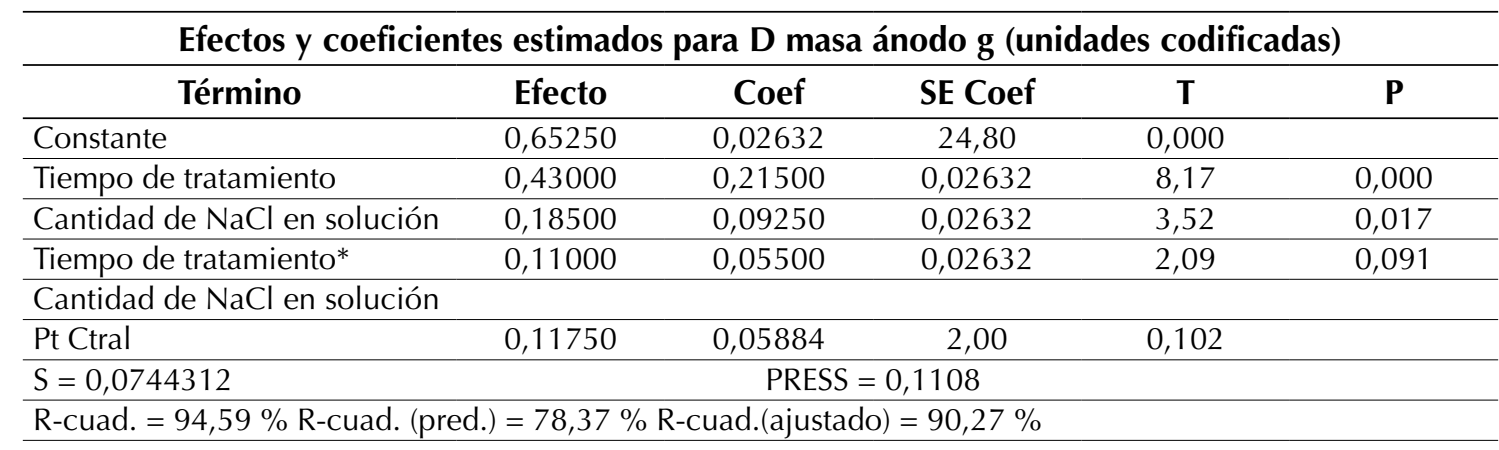


Es evidente el efecto significativo de ambos factores (figura 5), que coincide con lo reportado en literatura (Comninellis y Chen, 2010, Pepió y Gutiérrez-Bouzán, 2011).
Los residuales no presentan un comportamiento normal para esta variable respuesta (figura 6), pero esta variabilidad podría haberse corregido con un número mayor de réplicas.

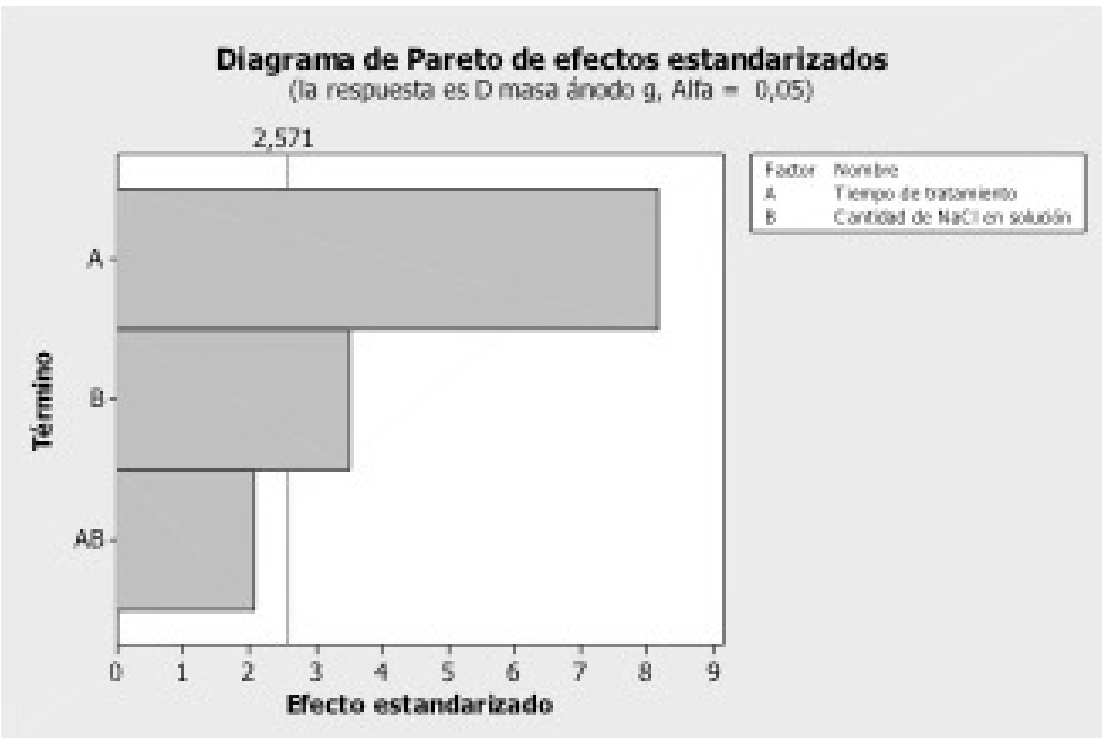

Figura 5. Pareto de efectos para disminución de masa del ánodo de sacrificio

Fuente: elaboración propia.

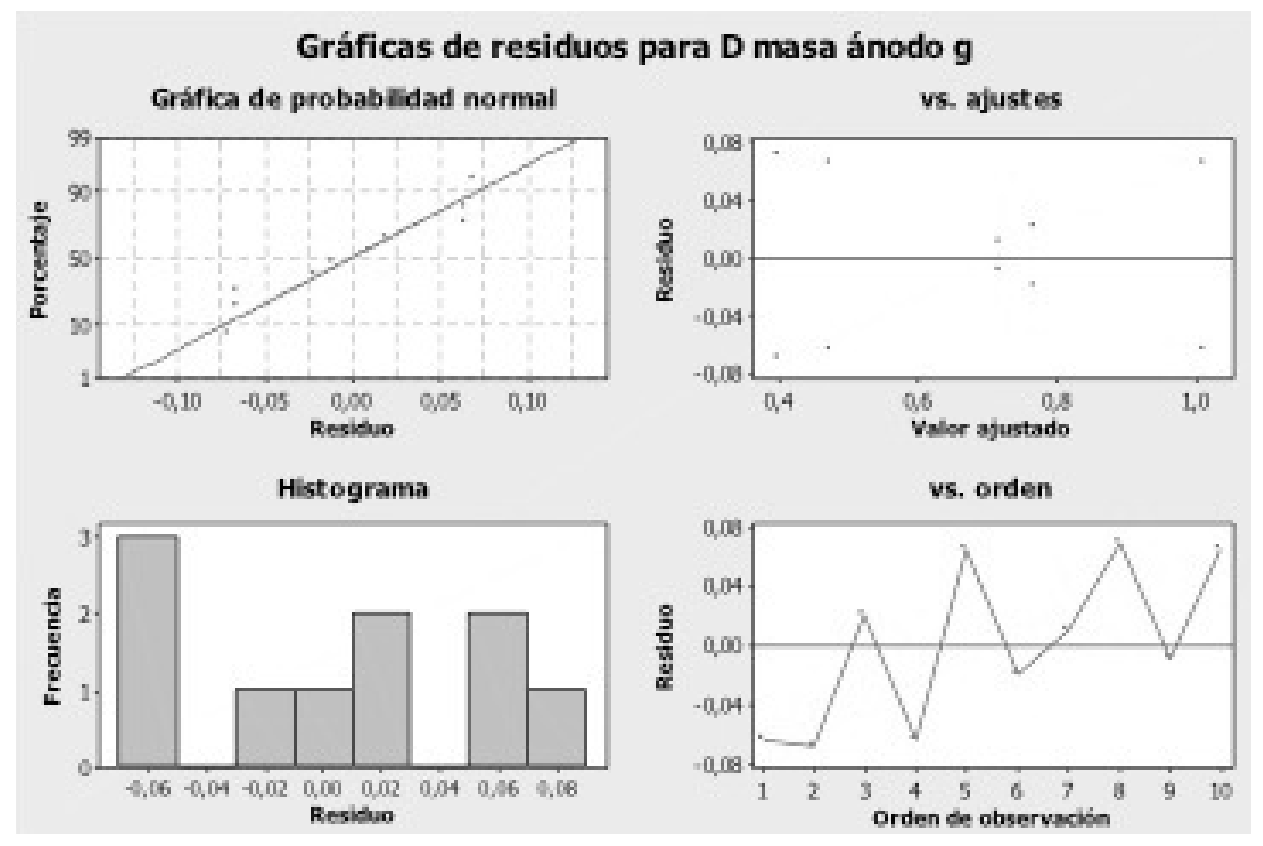

Figura 6. Gráfica de residuos para disminución de masa del ánodo de sacrificio

Fuente: elaboración propia. 


\section{CONCLUSIONES}

Los resultados de DQO no fueron los esperados debido a los porcentajes de remoción obtenidos en los niveles probados. Este hecho evidencia la posibilidad de que el proceso de descontaminación por electrocoagulación pueda ser reversible con el tipo de ánodos que se acondicionaron para este fin, ya que en los puntos experimentales donde se esperaba una lectura más baja de DQO se obtuvieron valores más altos.

Esto puede indicar que al desestabilizarse los flóculos; el aluminio proveniente del ánodo de sacrificio que se utilizó como agente coagulante en el agua tratada está siendo liberado; y al ser tan oxidable este metal, puede ser la causa de la contaminación de las lecturas de demanda química de oxígeno.

Un factor adicional que puede ser causante de la liberación del ion aluminio es la disminución del $\mathrm{pH}$ que no fue evaluado durante la etapa experimental.

Es recomendable, para estudios futuros, corroborar este hecho y acompañar el seguimiento del proceso de electrocoagulación con registros de $\mathrm{pH}$, para establecer si puede llegar a incidir en la eficiencia de esta técnica y, de ser necesario, utilizar soluciones buffer que permitan mantener estable el $\mathrm{pH}$.

El comportamiento esperado y la incidencia de los factores escogidos para el diseño pueden verse en la medida de la disminución de masa del ánodo de sacrificio.

\section{REFERENCIAS}

Bayram, E. \& Ayranci, E. (2010). Electrochemically Enhanced Removal of Polycyclic Aromatic Basic Dyes from Dilute Aqueous Solutions by Activated Carbon Cloth Electrodes. Environmental Science \& Technology Environ. Sci. Technol., 44 (16), 6331-6336.

Can, O. \& Bayramoglu, M. (2010). The Effect of Process Conditions on the Treatment of Benzoquinone Solution by Electrocoagulation. Journal of Hazardous Materials, 173 (1-3), 731-736.
Cañizares, P.; Gadri, A.; Lobato, J.; Nasr, B.; Paz, R.; Rodrigo, M. \& Saez, C. (2006). Electrochemical Oxidation of Azoic Dyes with Conductive-Diamond Anodes. Industrial \& Engineering Chemistry Research Ind. Eng. Chem. Res., 45 (10), 3468-3473.

Cañizares, P.; Martínez, F.; Lobato, J. \& Rodrigo, M. A. (2006) Electrochemically Assited Coagulation of Wastes Polluted with Eriochrome Black T. Industrial \& Engneering Chemistry Research Ind. Eng. Chem. Res., 45 (10), 3474-3480.

Cerqueria, A., Russo, C., \& Marques, M. R. C. (2008). Electroflocculation for Textile Wastewater Treatment. Brazilian Journal of Chemical Engineering, 26 (4), 659-668.

Chen, X., Chen, G., \& Yue, P. L. (2002). Novel Electrode System for Electroflotation of Wastewater. Environmental Science and Technology, 36 (4), 778-783.

Comninellis, C. \& Chen, G. (2010). Electrochemistry for the environment. Nueva York: Springer.

Daneshvar, N.; Ashassi-Sorkhabi, H. \& Tizpar, A. (2003). Decolorization of Orange II by Electrocoagulation Method. Separation and Purification Technology, 31 (2), 153-162.

Dávila, J. A., Machuca, F. \& Marrianga, N. (2008). Reducción de demanda química de oxígeno, carbono orgánico total y sólidos totales en vinazas mediante electro- flotación/oxidación. Revista de Ingeniería e Investigación, 29 (1), 35-38.

Dávila, J.; Machuca, F. \& Marrianga, N. (2010). Peroxidación y electroflotación de vinazas de destilaría. Rev. Ing., 32., 38-44.

Dirección de Agua Potable y Saneamiento Básico, Ministerio de Desarrollo Económico, República de Colombia (2000). Reglamento técnico del sector de agua potable y saneamiento básico, Sección II: Tratamiento de aguas residuales.

Gutiérrez, H. \& De la Vara, R (2004). Análisis y diseño de experimentos. México: McGraw-Hill/Interamericana Editores, S.A.

Hernlem, B. J., \& Tsai, L. S. (2000). Chlorine Generation and Disinfection by Electrocoagulation. Journal of Food Science, 65 (5), 834-837.

Modirshahla, N.; Behnajady, M. \& Kooshaiian, S. (2007). Investigation of the Effect of Different Electrode 
Connections on the Removal Efficiency of Tartrazine from Aqueous Solutions by Electrocoagulation. Dyes and Pigments, 74 (2), 249-257.

Mollah, M.; Morkovsky, P.; Gomes, J.; Kesmez, M.; Parga, J. \& Cocke, D. (2004). Fundamentals, Present and Future Perspectives of Electrocoagulation. Journal of Hazardous Materials, 114 (1-3), 199-210

Mu, Y., Rabaey, K., Rozendal, R., Yuan, Z., \& Keller, J. (2009). Decolorization of Azo Dyes in Bioelectrochemical Systems. Environmental Science \& Technology Environ. Sci. Technol., 43 (13), 5137-5143.

Norma Técnica Colombiana NTC 5167 de 2004.

Pepió, M, \& Gutiérrez-Bouzán, M. (2011). Empirical Models for the Decoloration of Dyes in an Electrochemical Batch Cell. Industrial \& Engineering Chemistry Research Ind. Eng. Chem. Res., 50(15), 8965-8972.

Peters, A., \& Freeman, H. S. (1995). Modern colorants: Synthesis and structure. Glasglow, Great Britain: Blackie Academic and Proffessional.

Petrinić, I.; Andersen, N.; Šostar-Turk, S.The Removal of Reactive Dye Printing Compounds Using Nanofiltration. Dyes and Pigments, 74 (3), 512-518.

Rojas, J. \& Giraldo, L. (2009a). Empleo del reactivo de Fenton para la degradación del colorante Tartrazina. Revista Lasallista de Investigación, 6 (1), 27-34.
Rojas, J. \& Giraldo, L. (2009b). Mineralizacion del colorante tartrazina mediante un proceso de oxidación avanzada. Revista Lasallista de Investigación, $6(2), 46-53$

Rojas, J.A.; Giraldo, L.G.; Ruiz, A.A.; López, C.A. \& Rivera, C. M. (2008). Degradación del colorante tartrazina mediante fotocatálisis heterogénea empleando lámpara de luz ultravioleta. Producción Más Limpia 3(2), 37-47.

Szpyrkowicz, L.; Cherbanski, R. \& Kelsall, G. (2005). Hydrodynamic Effects on the Performance of an Electrochemical Reactor for Destruction of Disperse Dyes. Industrial \& Engineering Chemistry Research Ind. Eng. Chem. Res., 44 (7) 2058-2068.

Szpyrkowicz, L.; Juzzolino, C.; Kaul, S.; Daniele, S. \& Faveri, M. (2000). Electrochemical Oxidation of Dyeing Baths Bearing Disperse Dyes. Industrial \& Engineering Chemistry Research Ind. Eng. Chem. Res., 39 (9), 3241-3248.

Szpyrkowicz, L. (2005). Hydrodynamic Effects on the Performance of Electro-coagulation/Electro-flotation for the Removal of Dyes from Textile Wastewater. Industrial \& Engineering Chemistry Research Ind. Eng. Chem. Res., 44 (20), 7844-7853. 
